\title{
Antigenic drift as a mechanism for tumor evasion of destruction by cytolytic $T$ lymphocytes
}

\author{
Xue-Feng Bai, Jinqing Liu, Ou Li, Pan Zheng, and Yang Liu \\ Division of Cancer Immunology, Department of Pathology and Comprehensive Cancer Center, \\ Ohio State University Medical Center, Columbus, Ohio, USA
}

\begin{abstract}
It is established that mutations in viral antigenic epitopes, or antigenic drifts, allow viruses to escape recognition by both Ab's and T lymphocytes. It is unclear, however, whether tumor cells can escape immune recognition via antigenic drift. Here we show that adoptive therapy with both monoclonal and polyclonal transgenic CTLs, specific for a natural tumor antigen, P1A, selects for multiple mutations in the P1A antigenic epitope. These mutations severely diminish $\mathrm{T}$ cell recognition of the tumor antigen by a variety of mechanisms, including modulation of MHC:peptide interaction and TCR binding to MHC:peptide complex. These results provide the first evidence for tumor evasion of $\mathrm{T}$ cell recognition by antigenic drift, and thus have important implications for the strategy of tumor immunotherapy.
\end{abstract}

J. Clin. Invest. 111:1487-1496 (2003). doi:10.1172/JCI200317656.

\section{Introduction}

It is well established that both neutralizing Ab's $(1,2)$ and cytotoxic $\mathrm{T}$ lymphocytes can select for viral escape variants in vivo $(3,4)$. This is usually achieved by mutations that result in replacement of one or more AAs within the antigenic epitopes, a process known as antigenic drift. A recent study revealed that virus-specific CD4 T cells can also select for viral escape variants in vivo (5). Much like viruses, tumors can also evade cytolytic T cells in vivo (6). The described mechanisms include immune ignorance $(7,8)$, induction of clonal anergy of tumor-specific T cells (9), downregulation of antigen presentation $(10,11)$, and loss of expression of tumor antigen (12). To our knowledge, it is still unclear whether mutations in tumor antigenic epitopes contribute to tumor evasion of the immune system in vivo.

Transgenic mice expressing $\mathrm{T}$ cell receptors for a single antigenic epitope play an instrumental role in establishing antigenic mutation as a mechanism for viral escape of $\mathrm{T}$ cell recognition $(3,5)$. We have recently produced a transgenic mouse line expressing a TCR specific for tumor antigen $\mathrm{P} 1 \mathrm{~A} 35-43$ presented by $\mathrm{H}-2 \mathrm{~L}^{\mathrm{d}}$ (P1CTL) (13) and have found that large unmodified tumors are highly resistant to therapy using the P1CTL $(14,15)$. In the process of studying the mechanism for resistance of large tumors, we have frequently observed

Received for publication December 18, 2002, and accepted in revised form March 6, 2003.

Address correspondence to: Yang Liu, Division of Cancer Immunology, Department of Pathology, Ohio State University

Medical Center, 129 Hamilton Hall, 1645 Neil Avenue, Columbus, Ohio 43210, USA. Phone: (614) 292-3054;

Fax: (614)688-8152; E-mail: liu-3@medctr.osu.edu.

Conflict of interest: The authors have declared that no conflict of interest exists.

Nonstandard abbreviations used: $\mathrm{T}$ cell receptor (TCR); transgenic T cells expressing the $\alpha$ and $\beta$ chains of TCR specific for tumor antigen $\mathrm{P} 1 \mathrm{~A} 35-43$ peptide presented by $\mathrm{H}-2 \mathrm{~L}^{\mathrm{d}}(\mathrm{P} 1 \mathrm{CTL})$; $\mathrm{PE}$, phycoerythrin. recurrences of tumors in mice that have responded favorably to therapy with high numbers of transgenic T cells. To analyze the mechanism of tumor evasion of $\mathrm{T}$ cell therapy, we systematically characterized the P1A antigen among the recurrent tumors. We uncovered a large collection of tumor variants with mutations within the P1A epitope. These mutations abolished $\mathrm{T}$ cell recognition of the tumor cells by modulating either peptide binding to the MHC molecules or, more importantly, the binding of the MHC:peptide complex to the TCR. These results demonstrate antigenic drift of tumor antigens as a mechanism for tumor evasion of CTL therapy in vivo.

\section{Methods}

Experimental animals. Transgenic mice expressing a TCR specific for the tumor antigen $\mathrm{H}-2 \mathrm{~L}^{\mathrm{d}}$ :P1A35-43 complex have been described (13). TCR transgenic mice were backcrossed with BALB/cByJ mice for at least nine generations before they were used for this study. $\mathrm{BALB} / \mathrm{c}$ mice with a targeted mutation of the RAG-2 gene were purchased from Taconic Farms (Germantown, New York, USA).

Production of TCR $\alpha$ chain-transgenic mice. The transgenic vector consisting of the $\alpha$ chain of the TCR from a P1A-reactive CTL clone has been described (13). The non-TCR-related vector sequence was removed prior to injection into fertilized eggs of FVB/N mice. Founder mice were screened by PCR using primers for the rearranged VJ segment of the transgenic vector and by flow cytometry of the $V \alpha 8$ chain on the surface of peripheral blood lymphocytes, as has been described (13).

Cell lines. $\mathrm{H}-2 \mathrm{~L}^{\mathrm{d}}$-transfected, transporter associated with antigen processing 2-deficient RMA-S cells were produced and kindly provided by Ted Hansen (Washington University, St. Louis, Missouri, USA) (16). The BALB/c plasmocytoma J558 transfected with a pSV 
vector (J558-Neo) has been described (14). To isolate tumor cells from ex vivo tissues, tumors were surgically removed and single-cell suspensions were prepared by grinding tumor tissues over two frosted glass slides. After removing the tumor debris, the viable cells were enriched by centrifugation through FicollPaque medium. The tumor cells were cultured in RPMI medium containing 5\% FCS for 1 week before they were used for flow cytometry and molecular analysis of the $P 1 A$ gene.

Adoptive transfer of purified transgenic T cells. Pools of spleen and lymph node cells from P1CTL-transgenic mice were incubated with a cocktail of mAb's (antiCD4 mAb GK1.5, anti-FcR mAb 2.4G2, and anti$\mathrm{CD} 11 \mathrm{c} \mathrm{mAb}$ N418). After removal of unbound mAb's, the cells were incubated with anti-Ig-coated magnetic beads (BioSource International, Keystone, Colorado, USA). The Ab-coated cells were removed by a magnet. The unbound cells consisted of more than $90 \%$ CD8 T cells, with no detectable CD4 T cells. The purified CD8 $\mathrm{T}$ cells $\left(5 \times 10^{6} / \mathrm{mouse}\right)$ were injected intravenously into mice bearing large tumors $(>1 \mathrm{~cm})$.

In other experiments, $2 \times 10^{7}$ spleen cells from either TCR $\alpha$ chain-transgenic mice (the F1 generation of a cross between transgenic founder and $\mathrm{BALB} / \mathrm{c}$ mice) or their nontransgenic littermates were adoptively transferred into mice with tumors greater than $1 \mathrm{~cm}$ in diameter. Tumor growth was determined by physical examination, while the number of tumor-reactive $\mathrm{T}$ cells was monitored by flow cytometry.

Molecular characterization of $P 1 A$ antigen from wild-type and CTL-resistant tumor cell lines. The expression of tumor antigen P1A was determined by RT-PCR using previously reported conditions (13). The primers used were 5'-GCCATGTCTGATAACAAGAAACCA-3' (forward) and $5^{\prime}$-TTGCAACTGCATGCCTAAGGTGAG-3' (reverse). The PCR products were analyzed using agarose gel electrophoresis followed by cloning and sequencing.

To analyze the genetic lesion of the P1A antigen, the genomic DNA was isolated from either ex vivo tumor cells or their subclones, which were obtained by limiting dilutions. The $P 1 A$ gene fragments were amplified by PCR using 5'-GCTAGCTTGCGACTCTACTCTTATCT- $3^{\prime}$ as the forward primer and $5^{\prime}$-TCCACATCCCTTTCATACTGCTCC- $3^{\prime}$ as the reverse primer. The PCR products were cloned and sequenced. In some experiments, the PCR products were analyzed by digestion with a panel of restriction enzymes, namely AvaII, Fnu4HI, HaeIII, and BbsI, which distinguish wild-type and mutant $P 1 A$ genes at positions 1 , $6,7,8$, and 9 .

Peptide synthesis. The wild-type and mutant P1A peptides, as well as the control $\mathrm{H}-2 \mathrm{~L}^{\mathrm{d}}$ binding peptide from murine cytomegalovirus. All peptides were synthesized by Research Genetics (Huntsville, Alabama, USA), dissolved in ethanol, and stored at $-20^{\circ} \mathrm{C}$.

Stabilization of cell-surface $H-2 L^{d}$ on RMA-S- $L^{d}$. RMA-S- $L^{d}$ cells were incubated with a given concentration of wildtype and mutant $\mathrm{P} 1 \mathrm{~A}$ peptides at $37^{\circ} \mathrm{C}$ for 16 hours in the presence of human $\beta_{2}$-microglobulin $(2.5 \mu \mathrm{g} / \mathrm{ml}$; Sigma-Aldrich, St. Louis, Missouri, USA). Cell-surface $\mathrm{H}-2 \mathrm{~L}^{\mathrm{d}}$ was analyzed by flow cytometry.

Flow cytometry. Cell-surface expression of $\mathrm{H}-2 \mathrm{~L}^{\mathrm{d}}$ was detected using biotinylated mAb 28-14-8 (BD Pharmingen, San Diego, California, USA) followed by phycoerythrin-labeled (PE-labeled) streptavidin. To determine the binding of $\mathrm{H}-2 \mathrm{~L}^{\mathrm{d}}$ :peptide complex to transgenic $\mathrm{T}$ cells, we used the H-2 $\mathrm{L}^{\mathrm{d}}$ :Ig dimer (purchased from BD Pharmingen) according to the manufacturer's instructions. Briefly, $3 \mu \mathrm{g}$ of peptides were incubated with 4 $\mu \mathrm{g}$ of $\mathrm{H}-2 \mathrm{~L}^{\mathrm{d}}: \mathrm{Ig}$ and $\beta_{2}$-microglobulin complex at $4^{\circ} \mathrm{C}$ for 48 hours in a total volume of $200 \mu$ l. PE-conjugated rat anti-mouse IgG1 mAb was added to the solution 1 hour before it was used to stain spleen cells from transgenic mice whose $T$ cells expressed the TCR specific for the H-2 $L^{d}: P 1 A$ peptide complex. After washing away the unbound complex, the spleen cells were fixed with $1 \%$ paraformaldehyde in PBS and analyzed by flow cytometry.

Proliferation assay. To measure proliferation of transgenic T cells, spleen T cells $\left(10^{5} /\right.$ well) were cultured in 96-well plates in the presence of given concentrations of peptides. After 66 hours of culture, $1.25 \mu \mathrm{Ci} /$ well of ${ }^{3} \mathrm{H}$-thymidine was added. The cultures were harvested 12 hours later, and the incorporation of ${ }^{3} \mathrm{H}-\mathrm{TdR}$ was measured and used as an indicator of $\mathrm{T}$ cell proliferation.

Cytotoxicity assay. Transgenic spleen cells were stimulated with the P1A peptide $(0.1 \mu \mathrm{g} / \mathrm{ml})$ for 4 days and used as effectors. As targets, we used ${ }^{51} \mathrm{Cr}$-labeled P388D1 $\left(\mathrm{H}-2^{\mathrm{d}}\right)$ cells. Various concentrations of peptides were added to the target cells immediately before the addition of the effector $T$ cells. In the competition assay, the peptides added were a mixture of a given concentration of competing peptides and $10 \mathrm{ng} / \mathrm{ml}$ of the wildtype P1A peptide. The effector $\mathrm{T}$ cells and the targets were coincubated for 6 hours, and the percentages of specific lysis were calculated based on the following formula: specific lysis $\%=100 \times\left(\mathrm{cpm}_{\text {sample }}-\mathrm{cpm}_{\text {medium }}\right) /$ $\left(\mathrm{cpm}_{\max }-\mathrm{cpm}_{\text {medium }}\right)$.

\section{Results}

Monoclonal transgenic $T$ cell therapy of large tumors in vivo selects for $T$ cell-resistant tumor variants. It is generally accepted that large tumors are highly resistant to CTL therapy. To analyze the mechanism responsible for such resistance, we transplanted the plasmocytoma J558-Neo into syngeneic $R A G-2^{-/-} \mathrm{BALB} / \mathrm{c}$ mice. Once the tumors had reached a size of $1.2-1.4 \mathrm{~cm}$ in diameter, we adoptively transferred purified P1CTLs into tumor-bearing mice. At this point, the tumors would grow progressively in the untreated mice and euthanasia would be necessary within 1 week (14). Therapy with P1CTLs would prolong the life of the mice, although curing of large tumors was not achieved (14). As shown in Figure 1a, a reduction of more than $60 \%$ in tumor volume was observed within 1 week of $\mathrm{T}$ cell transfer. Histological examination revealed that the overwhelming majority of the tumor mass consisted 
of necrotic tumor cells, although a small number of viable tumor cells was found (data not shown). However, tumor shrinkage stalled in the next 2 weeks. Starting from the third week, tumors resurged in all mice treated. We then started a new round of transfer of transgenic $\mathrm{T}$ cells. However, the tumors were no longer responsive to $\mathrm{T}$ cell treatment.

We isolated tumor cells from two of four mice that had received two rounds of CTL therapy and tested their susceptibility to cytolysis by activated transgenic $\mathrm{T}$ cells in vitro. The data in Figure $1 \mathrm{~b}$ indicate that while parental tumor cells were readily killed by P1CTLs, those isolated from the two T cell-resistant tumors were completely refractory to treatment. RT-PCR analysis indicated that transcripts of tumor antigen P1A were expressed in both tumor lines (Figure 1c). Moreover, both lines expressed cell-surface $\mathrm{H}-2 \mathrm{~L}^{\mathrm{d}}$ at levels comparable to levels found on J558-Neo cells (Figure 1d). These results demonstrate that therapy with P1CTLs selects for tumor variants that are resistant to P1CTLs, and at least for the two resistant tumor lines tested here, resistance is not due to the lack of cell-surface MHC or loss of expression of the $P 1 A$ gene.

Molecular lesions of P1A genes in tumor cells that evaded monoclonal T cell therapy. We isolated and sequenced five clones of P1A cDNA from each PCR product in Figure 1c. All cDNA clones from the J558-Neo tumors had the wild-type P1A sequence (Figure 2a), while those recovered from the CTL-resistant tumor cell lines harbored mutations within the P1A epitope, the nine-AA peptide corresponding to positions 35-43 of the predicted P1A protein (17). Four of five clones isolated from Tum 1 had a mutation that changed the AA sequence at position $6(\mathrm{~W} \rightarrow \mathrm{R}$, due to a point mutation of $\mathrm{T} \rightarrow \mathrm{C})$ of the P1A epitope, herein termed P1A(6R). The other had a mutation that altered the AA sequence at position $9(\mathrm{~F} \rightarrow \mathrm{L}$, also due to a $\mathrm{T} \rightarrow \mathrm{C}$ point mutation), hereby termed $\mathrm{P} 1 \mathrm{~A}(9 \mathrm{~L})$. All five clones from Tum 2 were P1A(6R).

We carried out two types of experiments to identify the genetic lesions in the P1A gene in the two ex vivo tumor cell lines. First, using limiting dilutions, we obtained more than 30 clones from each of the parental J558-Neo, Tum 1, and Tum 2 cell lines, and then analyzed these for the presence of mutations at P6 and P9. Based on the mutations identified in the cDNA clones, we identified two restriction enzymes that can recognize the mutations. As indicated in Figure $2 b$, a mutation in the $\mathrm{P} 6$ position $(\mathrm{T} \rightarrow \mathrm{C})$ rendered the $\mathrm{P} 1 \mathrm{~A}$ product susceptible to digestion by Fnu4HI, while a mutation in the P9 position $(\mathrm{T} \rightarrow \mathrm{C})$ allowed recognition by AvaII. We therefore analyzed the genomic PCR products from multiple cell clones from parental J558-Neo, Tum 1, and Tum 2 cells for the presence of genetic lesions. As shown in Figure 2b, no PCR product from any of the 37 clones from J558-Neo cells harbored the P6 and P9 mutations. Further sequence analysis of multiple clones uncovered no mutations in the antigenic epitopes of the parental J558-Neo tumor cells, including those that were passed either in vitro or in vivo in untreated $R A G-2^{-/-}$mice (data not shown). Thus, in the absence of a strong immune response, the P1A antigenic epitope remains unchanged.

The clones isolated from the Tum 1 line can be divided into four groups ( $b$, lower panel). Group $1(3 / 35)$ harbored a mutation at P9. Group $2(3 / 35)$ appeared to be heterogeneous at position $\mathrm{P} 9$, while having no mutation at P6. Our analysis of the RNA transcripts derived from a representative clone from this group by RT-PCR revealed that the only form expressed was P1A(9L) (c). Thus the other potential allele was inactivated by some

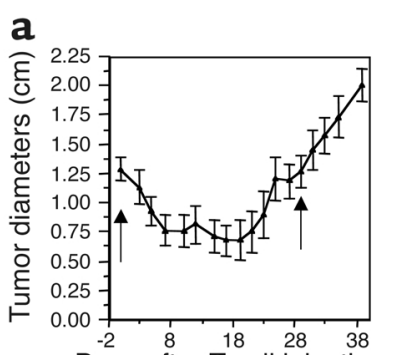

d
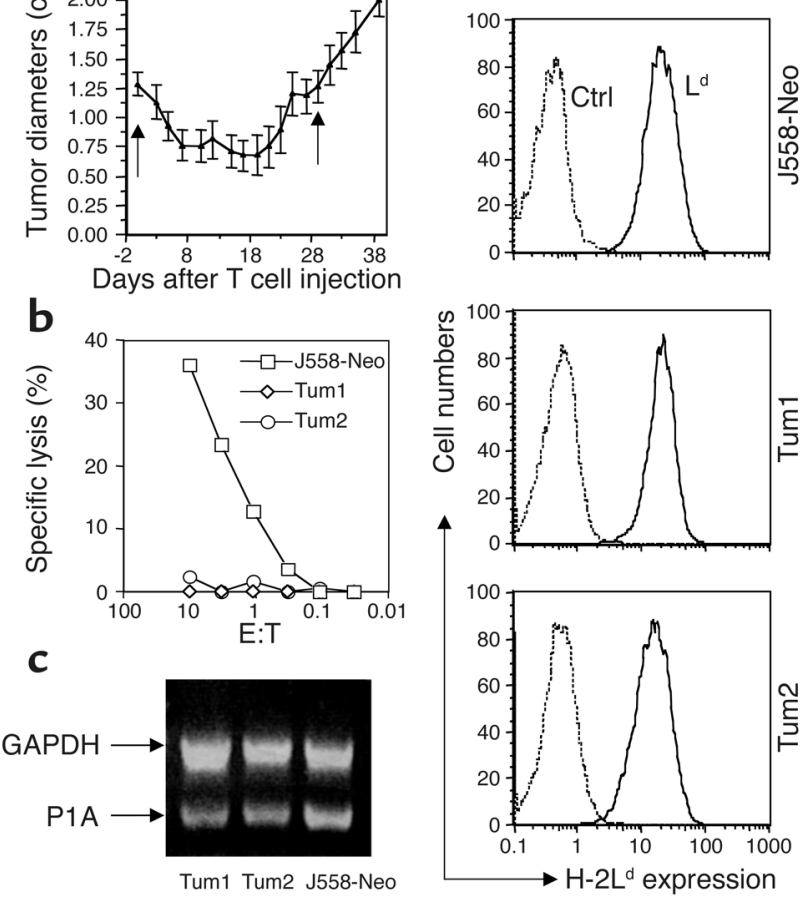

Figure 1

Adoptive therapy with P1A-specific transgenic $T$ cells selects for $T$ cell-resistant tumor cells. (a) Treatment of large P1A-expressing tumors with transgenic $T$ cells results in rapid shrinkage of tumors, followed by stagnation and resurgence of tumor growth. J558-Neo tumor cells $\left(5 \times 10^{6}\right)$ were injected subcutaneously into RAG-2-/- BALB/c mice. Two to three weeks later, when the tumors reached $1.2-1.4 \mathrm{~cm}$ in diameter, purified transgenic T cells $\left(5 \times 10^{6} /\right.$ mouse $)$ were injected intravenously. The treatment was repeated on day 31 when the tumors had returned to their pretreatment size, as indicated by arrows. Mice were euthanized on day $39(n=4)$. (b) Tumor cells isolated after two treatments were resistant to cytolysis by activated P1A-specific $T$ cells. Tumor cells were isolated from two different mice and were compared with their parental tumor J558-Neo cells for their susceptibility to cytolysis in a 6-hour cytotoxicity assay. Data shown are representative two experiments. A, Avall; F, Fnu4HI; U, uncut. (c) Expression of P1A was not lost in the P1CTL-resistant tumor cells. First-strand DNA was generated from total RNA using reverse transcriptase and amplified with primers specific for either GAPDH (700-bp product) or P1A (500-bp product). (d) Normal expression of $\mathrm{H}-2 \mathrm{~L}^{\mathrm{d}}$ on the surface of $\mathrm{CTL}$-resistant tumor cells. Histograms depict binding of second-step reagent (dotted lines) or $\mathrm{H}-2 \mathrm{~L}^{\mathrm{d}}$-specific $\mathrm{mAb}$ followed by the second-step reagent (solid lines). $\mathrm{E} / \mathrm{T}$, effector to target ratio. 
a Sequence chromatogram WT (P1A) $\begin{array}{lll}\text { CTGCCTTATCTAGGGTGG TGG } \\ 20 & 230 & 240\end{array}$

WWMMWWMWWMWM P6R LPYLGWLVF

C TGCCTTATCTAGGGCGGC TGGT CTTC $220230+240$

NAMMNWWMW Mharh LPYLGRLVF

P9L

CTGCCTTATCTAGgGtgGe TGgTCC TC

$230240 \quad 250$

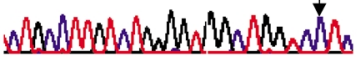
LPYLGWLVL

P7P $290 \quad 300 \quad \downarrow 310$

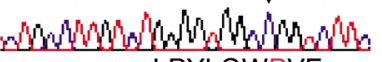
LPYLGWPVF

P8G

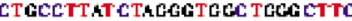

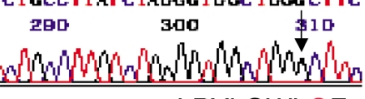
LPYLGWLGF b Restriction enzyme mapping
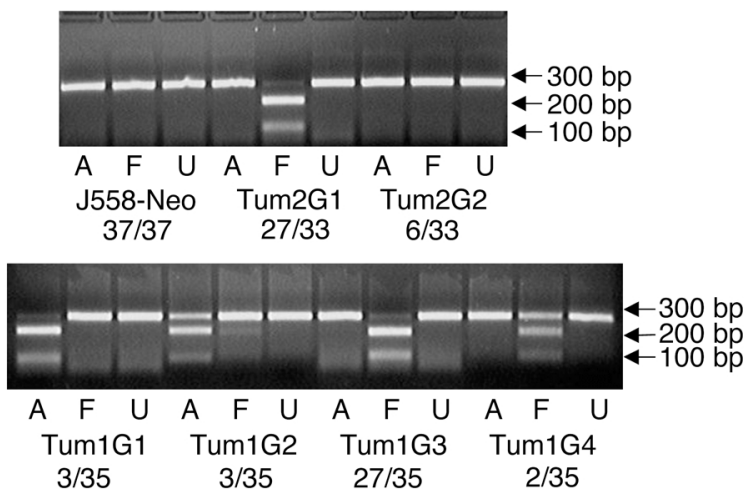

C

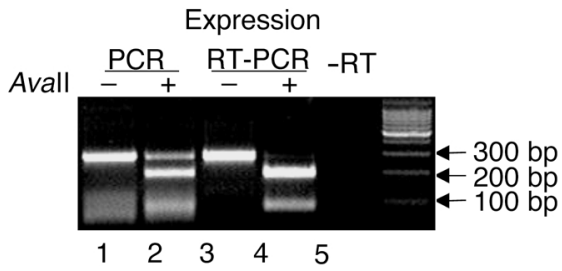

\section{Figure 2}

Molecular lesions in the P1A gene of the recurrent tumors. (a) Chromatograms of sequencing reactions of both cDNA and genomic PCR products. Mutations in nucleotides are indicated by arrows, while the replaced AAs are colored red. (b) Restriction enzyme mapping of the subclones isolated either from the parental J558-Neo cells or from the recurrent tumor cell lines Tum 1 and Tum 2. Avall recognizes GGT(A)CC, which can be generated in the mutant P1A(9L); Fnu4HI is specific for GCNGC, which is present in P1A(6R). The two enzymes failed to identify any mutation in 37 clones of J558-Neo tumor cells. However, they divided the Tum 1 clones into four groups and Tum 2 clones into two groups (shown in figure as G1, G2, G3, and G4). The proportion of clones within each group is presented underneath each photograph. All suggested mutations of Tum 2 and Tum 1 and lack of mutation in J558-Neo clones were confirmed by DNA sequencing. DNA sequencing also revealed that the uncut Tum 2 G2 subclones harbored two independent mutations, P1A(7P) and P1A(8G). (c) Expression of the $P 1 A$ gene in the Tum 1 G2 clones. RNA was isolated and used for RT-PCR (lane 3 and lane 4). No product was observed when the reverse transcriptase (RT) was not used (lane 5). As a comparison, PCR products from genomic DNA were also produced (lane 1 and lane 2). Note that while the genomic DNA is apparently heterozygous at P9, only P1A(9L) RNA is observed in the tumor cells. WT, wild type.

yet-unidentified mechanism. Group 3 (27/35), which represented the majority of the clones isolated from Tum 1, had a mutation at P6. Group 4 members $(2 / 35)$ are heterogeneous at P6 while having a wild-type sequence at $\mathrm{P} 9$. The cells in this group were lost prior to RNA analysis, and it is therefore unclear whether the apparent wild-type allele was also inactivated. Tum 2 clones can be divided into two groups, the majority of them (27/33) having a mutation at the P6 position, and the minority of them $(6 / 33)$ harboring no mutation in either of these two positions (Figure $2 b$, upper panel). We also carried out extensive sequence analysis of the PCR products from Tum 1 and Tum 2. Our sequencing confirmed the mutations identified by enzymatic digestion. In addition, analysis of Tum 2 clones in which P1A mutations were not identified by enzymatic digestion revealed additional lesions in the antigenic epitope. Of the six clones from Tum 2 with no mutation at $\mathrm{P} 6$ and $\mathrm{P} 9$, one had a mutation at $\mathrm{P} 7(\mathrm{~T} \rightarrow \mathrm{C}$, resulting in an $\mathrm{L} \rightarrow \mathrm{P}$ substitution), while five others harbored a mutation at $P 8(T \rightarrow G$, leading to a $V \rightarrow G$ replacement), as shown in Figure $2 \mathrm{a}$.

Immunological basis of tumor evasion of monoclonal $T$ cell therapy. To test immunological consequences of these mutations, wild-type and mutant $\mathrm{P} 1 \mathrm{~A}$ peptides were synthesized and tested for their recognition by P1Aspecific transgenic T cells. An $\mathrm{H}-2 \mathrm{~L}^{\mathrm{d}}$ binding peptide from murine cytomegalovirus was used as control (Figure $3 \mathrm{a})$. As shown in Figure 3b, the mutant P1A(9L) peptide was 100-fold less potent than the wild type in inducing proliferation of transgenic $\mathrm{T}$ cells, while mutant P1A(7P) was about 1,000-fold less potent. Mutants P1A(6R) and P1A(8G) failed to induce any detectable $T$ cell proliferation.

In a CTL assay to measure cytolysis of P388D 1 target cells (Figure $3 \mathrm{c}$ ), the $\mathrm{P} 1 \mathrm{~A}(9 \mathrm{~L})$ peptide was at least 10- to 100 -fold less efficient than wild-type peptide, while P6 and P8 displayed a 10,000-fold reduction in inducing cytolysis of target cells. The most effective mutation was P1A $(8 \mathrm{G})$, which completely abolished recognition by P1CTLs.

Low-avidity TCR ligands can be TCR antagonists (18, 19). To test whether the mutant peptides are antagonists for $\mathrm{P} 1 \mathrm{~A}$, we incubated varying concentrations of the mutant or wild-type $\mathrm{P} 1 \mathrm{~A}$ peptides, or unrelated $\mathrm{H}$ $2 \mathrm{~L}^{\mathrm{d}}$-binding peptides, with the target cells in the presence of a fixed concentration of $\mathrm{P} 1 \mathrm{~A}$ peptide, and tested target-cell susceptibility to P1A-specific CTLs. As 
a Peptide sequence

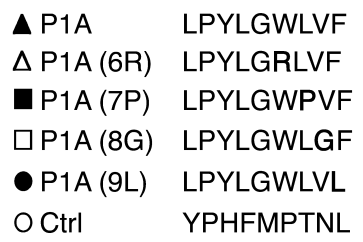

b

Proliferation
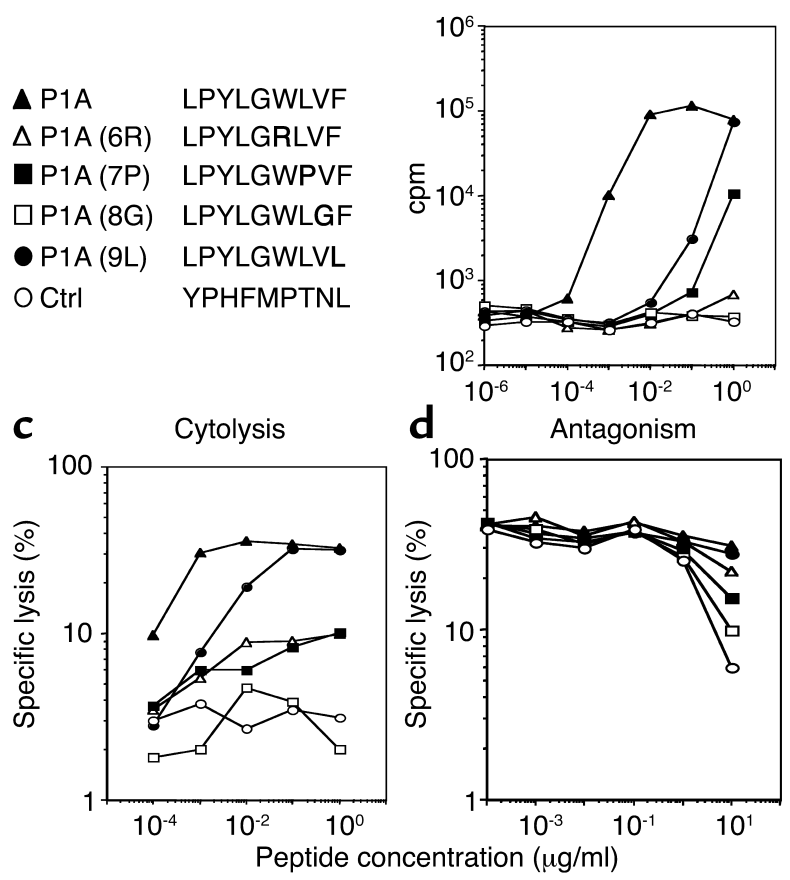

shown in Figure 3d, P1A(9L) caused essentially no inhibition of P1A-mediated cytolysis. Moderate inhibition by $\mathrm{P} 1 \mathrm{~A}(6 \mathrm{R}), \mathrm{P} 1 \mathrm{~A}(7 \mathrm{P})$, and $\mathrm{P} 1 \mathrm{~A}(8 \mathrm{G})$ was observed only when the mutant peptide was present at a 1,000-fold higher concentration than the P1A peptide. However, since the mutant peptides were significantly less potent than the unrelated peptide, which had no measurable avidity to TCR (see below), the observed inhibition by these mutant peptides was likely due to competition for $\mathrm{H}-2 \mathrm{~L}^{\mathrm{d}}$ binding rather than to its antagonism to TCR. Thus, these mutant peptides had no demonstrable antagonistic activity. Regardless of which mutations they harbored, all mutant J558-Neo clones were resistant to cytolysis by activated P1CTLs (Figure 4). Thus, under physiological conditions in which the antigenic peptides were produced at limiting doses, partial inactivation was sufficient to prevent $\mathrm{T}$ cell recognition of the tumor cells.

In theory, reduction of $\mathrm{T}$ cell recognition of the antigenic peptide can be attributed to either reduced peptide binding to $\mathrm{MHC}$ or reduced recognition of the MHC:peptide complex by the TCR. We determined the binding of wild-type and mutant peptides to $\mathrm{H}-2 \mathrm{~L}^{\mathrm{d}}$ using a classic $\mathrm{H}-2$ stabilization assay with $\mathrm{L}^{\mathrm{d}}$-transfected RMA-S cells (16). The results are summarized in Figure 5. Interestingly, although P6 was not considered an anchor residue for $\mathrm{H}-2 \mathrm{~L}^{\mathrm{d}}$-bound peptide, we found that $\mathrm{P} 1 \mathrm{~A}(6 \mathrm{R})$ was somewhat less potent than the wildtype peptide in stabilizing $\mathrm{H}-2 \mathrm{~L}^{\mathrm{d}}$. This is perhaps due to the positive charge introduced by the mutation. Similarly, replacing one permissible anchoring residue $(F)$ with another (L) at P9 also had a moderate effect on peptide binding to $\mathrm{H}-2 \mathrm{~L}^{\mathrm{d}}$. As expected, a control peptide from murine cytomegalovirus that had L at P9 also

\section{Figure 3}

Mutations in the P1A epitope abolished or drastically reduced T cell recognition of the antigen. (a) The AA sequences of the peptides used in the study, with the altered AA shown in bold. (b) Proliferation of transgenic $T$ cells in response to either wild-type or mutant $\mathrm{P} 1 \mathrm{~A}$ peptide. (c) Cytolysis of $\mathrm{P} 388 \mathrm{D} 1$ target cells pulsed with given concentrations of wild-type, mutant, or unrelated control $\mathrm{H}-2 \mathrm{~L}^{\mathrm{d}}$ binding peptides. $E / T=10$. (d) P1A mutants lack antagonistic activity for the P1CTL. Activated P1A-specific T cells were used as effectors. The target cells were preincubated with a mixture of wild-type P1A $(10 \mathrm{ng} / \mathrm{ml})$ and the indicated concentrations of mutant or wildtype $\mathrm{P} 1 \mathrm{~A}$ or control peptides. $\mathrm{E} / \mathrm{T}=10$. Data shown were from a 6-hour ${ }^{51} \mathrm{Cr}$-release assay and are representative of three to five independent experiments. Ctrl, control.

reduced binding to $\mathrm{H}-2 \mathrm{~L}^{\mathrm{d}}$. Surprisingly, mutations in P7 and P8 increased peptide binding to H-2 $\mathrm{L}^{\mathrm{d}}$. All of the described alterations were statistically significant: $\mathrm{P} 1 \mathrm{~A}$ versus $\mathrm{P} 1 \mathrm{~A}(6 \mathrm{R}), P \leq 0.0001$; $\mathrm{P} 1 \mathrm{~A}$ versus $\mathrm{P} 1 \mathrm{~A}(7 \mathrm{P})$, $P=0.0492 ; \mathrm{P} 1 \mathrm{~A}$ versus $\mathrm{P} 1 \mathrm{~A}(8 \mathrm{G}), P=0.0316$; $\mathrm{P} 1 \mathrm{~A}$ versus P1A(9L), $P=0.025$.

To directly measure the effect of these mutations on $\mathrm{T}$ cell recognition of the $\mathrm{H}-2 \mathrm{~L}^{\mathrm{d}}$ :peptide complex, we loaded H-2 ${ }^{\mathrm{d}} \mathrm{rg}$ with a 166 -fold excess of peptides and tested their binding to P1CTL. As shown in Figure 6a, in the spleen of the TCR transgenic mice, about 94-99\% of CD8 T cells expressed high levels of the transgenic $\alpha$ chain (no $A b$ against $V \beta 1$ is available). About the same percentage of CD8 T cells were stained by the $\mathrm{H}-2 \mathrm{~L}^{\mathrm{d}}: \mathrm{P} 1 \mathrm{~A}$ dimer. In contrast, only about $0.1 \%$ of CD8 T cells were stained by the control H-2Ld:peptide dimer. These results confirmed both the sensitivity and the specificity of the dimer binding assay. As shown in Figure 6b, both P1A(6R)- and P1A(9L)-loaded $\mathrm{H}-2 \mathrm{~L}^{\mathrm{d}}$ gave significant, albeit reduced, binding to the

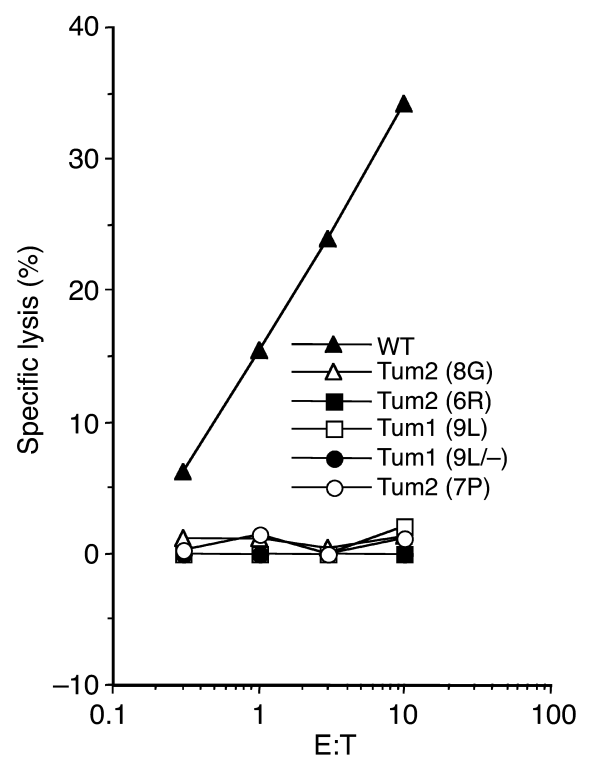

Figure 4

Susceptibility of wild-type, Tum 1, and Tum 2 subclones to P1A-reactive CTLs. The genotype of each clone is marked. Data shown are representative of two independent experiments. 

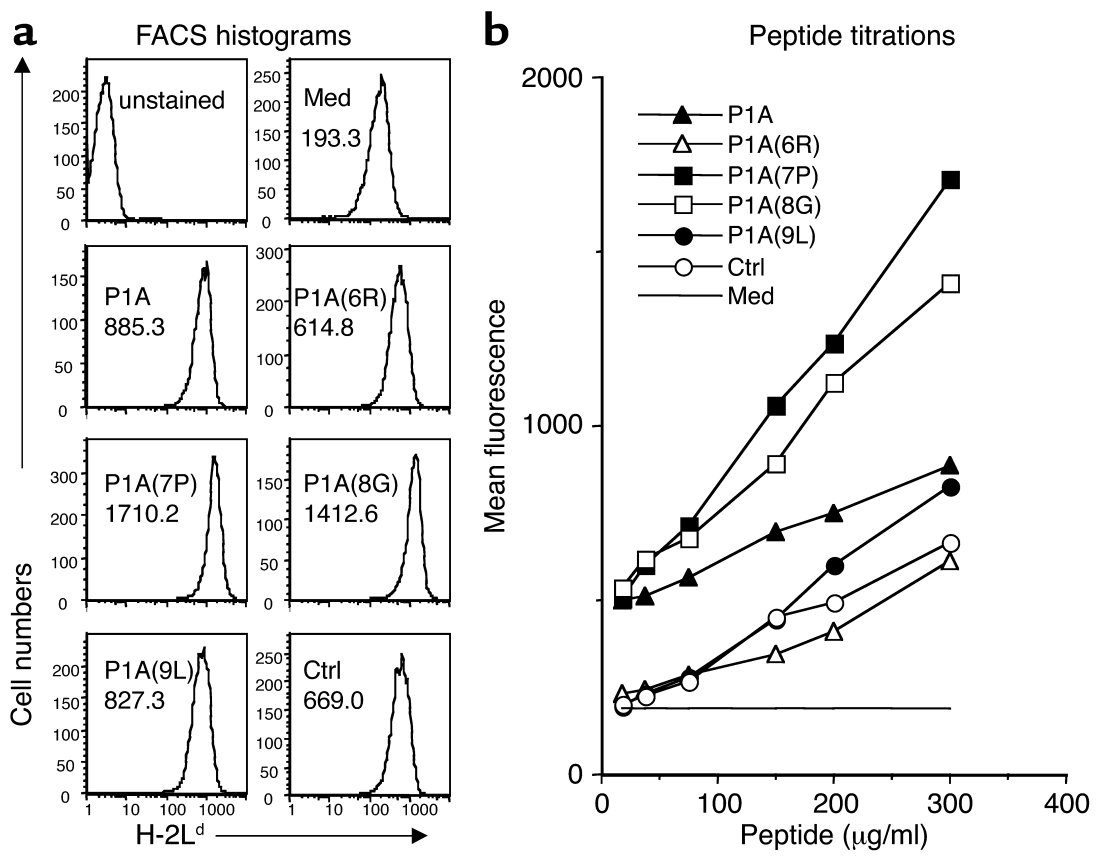
Figure 5
Peptide binding to $\mathrm{H}-2 \mathrm{~L}^{\mathrm{d}}$. After over night incubation with $\beta_{2}$-microglobulin and given concentrations of peptides, $\mathrm{H}-2 \mathrm{~L}^{\mathrm{d}}$-transfected RMA-S cells were stained with $\mathrm{H}-2 \mathrm{Ld}^{\mathrm{d}}$-specific mAb. (a) Representative FACS histograms. Num- bers in the panels are mean fluorescence intensities. (b) Quantitative comparison of peptide:H-2 $\mathrm{L}^{d}$ interaction. Data shown are representative of two to four independent experiments. Statistical sig- nificance was determined by paired $t$ test. Med, medium.

transgenic $\mathrm{T}$ cells. A much-reduced binding was observed with $\mathrm{H}-2 \mathrm{~L}^{\mathrm{d}} \mathrm{P} 1 \mathrm{~A}(7 \mathrm{P})$, while $\mathrm{H}-2 \mathrm{~L}^{\mathrm{d}}: \mathrm{P} 1 \mathrm{~A}(8 \mathrm{G})$ did not bind to P1CTLs. Titration of the MHC:peptide complex revealed that mutations $\mathrm{P} 1 \mathrm{~A}(6 \mathrm{R})$ and $\mathrm{P} 1 \mathrm{~A}(9 \mathrm{~L})$ reduced the avidity between the TCR and the MHC peptide by about two- to fourfold; while $\mathrm{H}-2 \mathrm{~L}^{\mathrm{d}}: \mathrm{P} 1 \mathrm{~A}(7 \mathrm{P})$ showed a more than 30 -fold reduction in binding to P1CTLs (Figure 6c). The reductions caused by all mutations were statistically significant (P1A versus $\mathrm{P} 1 \mathrm{~A}(6 \mathrm{R})$, $P=0.035$; $\mathrm{P} 1 \mathrm{~A}$ versus $\mathrm{P} 1 \mathrm{~A}(7 \mathrm{P}), P=0.03$; $\mathrm{P} 1 \mathrm{~A}$ versus $\mathrm{P} 1 \mathrm{~A}$ (8G), $P=0.0366$; $\mathrm{P} 1 \mathrm{~A}$ versus $\mathrm{P} 1 \mathrm{~A}(9 \mathrm{~L}), P=0.0398)$.

Polyclonal T cells with a transgenic TCR $\alpha$ chain from a $P 1 A$-reactive CTL clone selected antigenic variants in vivo. To extend our observations from mice with essentially monoclonal $\mathrm{T}$ cells, we produced a transgenic mouse line with the TCR $\alpha$ chain isolated from a P1Areactive CTL clone. Since no transgenic $\beta$ chain is present, the transgenic $\alpha$ chain must pair with endogenous $\beta$ chain to produce the cell-surface TCR/CD3 complex. As shown in Figure $7 \mathrm{a}$, in the $\mathrm{F} 1$ generation of transgenic founder and $\mathrm{BALB} / \mathrm{c}$ mice, more than $90 \%$ of the T cells expressed the transgenic TCR $\alpha$ chain, as revealed by a Va8-specific mAb. As a result, about $0.3 \%$ of CD8 T cells from TCR $\alpha$ chaintransgenic mice bound to the $\mathrm{H}-2 \mathrm{~L}^{\mathrm{d}}: \mathrm{P} 1 \mathrm{~A}$ complex (Figure 7a, lower panel), while no binding was seen in CD8 $\mathrm{T}$ cells from nontransgenic littermates (data not shown). To test whether $\mathrm{T}$ cells expressing the transgenic TCR $\alpha$ chain can cause rejection of large tumors, we adoptively transferred spleen cells from transgenic mice and their nontransgenic littermates into $R A G-2^{-/-}$mice bearing large tumors. As shown in Figure $7 \mathrm{~b}$, the transgenic spleen cells, but not those from their nontransgenic littermates, caused rapid shrinkage of tumors. However, essentially all tumors recurred within a 4-week period. The tumor rejection response was associated with significant expansion of P1A-reactive CD8 T cells, as $10-70 \%$ of CD8 T cells in tumor-bearing mice were $\mathrm{H}-2 \mathrm{~L}^{\mathrm{d}}$ :P1A-specific (Figure $7 \mathrm{c}$ ). We also analyzed the $V \beta$ usage of the CD8 T cells that reacted to $\mathrm{H}-2 \mathrm{~L}^{\mathrm{d}}$ :PIA complex by flow cytometry using most of the anti-V $\beta$ mAb's available. Our data demonstrate that, while the V $\beta$ usage for the majority of the $L^{d}$ :PIA-reactive $T$ cells had not been identified due to limitation of mAb's, cells expressing $V \beta 3, V \beta 4, V \beta 6, V \beta 8, V \beta 10, V \beta 11, V \beta 12$, and $V \beta 13$ can be found among the $L^{\mathrm{d}}$ :PIA-reactive population (data not shown).

To determine whether antigenic variants can arise in mice with polyclonal P1A-reactive T cells, we isolated the genomic DNA from two recurrent tumors. The P1A gene fragments were amplified and then subjected to digestion by a panel of three restriction enzymes. Fnu $4 \mathrm{HI}$ is specific to GCNGC and recognizes mutant P1A(6R). HaeIII, with a specificity for GGCC, can recognize mutations leading to $\mathrm{L} \rightarrow \mathrm{P}$ replacements at either P7 or P1. Any change in the six nucleotides encoding P8 and P9 will result in loss of digestion by BbsI, which recognizes GTCTTC. As shown in Figure $8 \mathrm{a}$, the P1A in tumors from nontransgenic T cell-treated mice was fully susceptible to $B b s$ I while being resistant Fnu4HI and HaeIII. Thus, in the absence of antigenspecific T cells, no mutations in the P1A epitope could be identified by restriction enzyme mapping. In contrast, the tumor cells recovered from mice treated with TCR $\alpha$ chain-transgenic T cells yielded P1A products that were partially resistant to $B b s$ I and partially susceptible to Fnu4HI. Thus, a substantial number of tumor cells harbored the mutation P1A(6R). To determine the mutations that rendered resistance to $B b s \mathrm{I}$, we cloned the PCR products and sequenced three of the clones that were resistant to BbsI. Two clones had a 


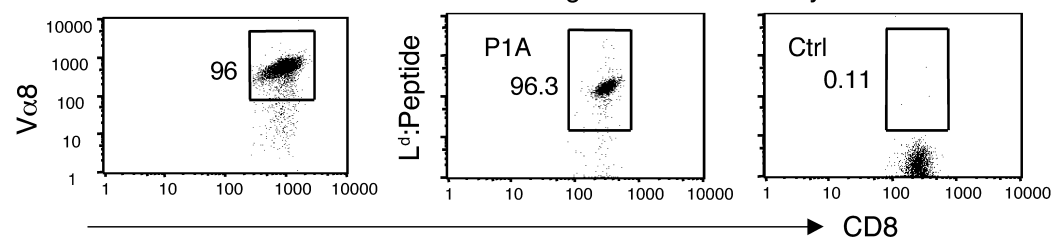

b
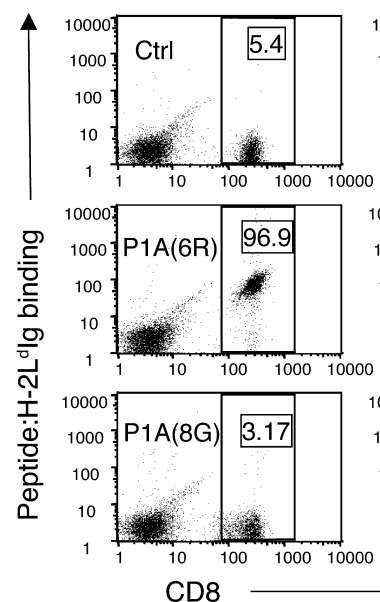

Dimer binding
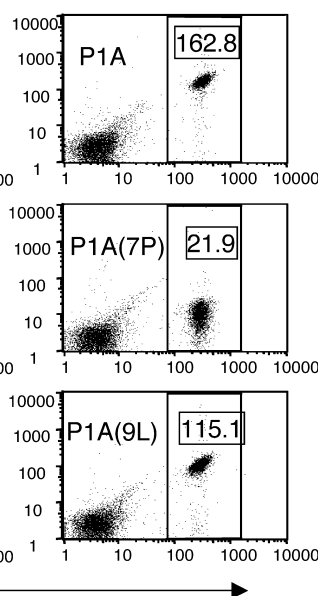

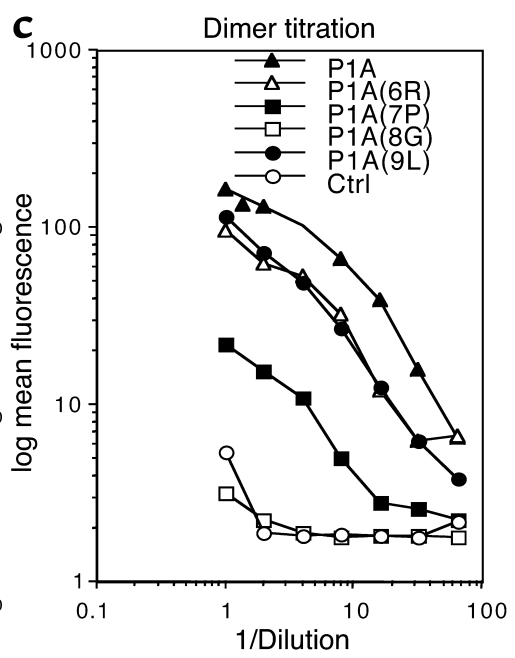

Figure 6

The effect of peptide alteration on the binding of P1A-specific T cells to the $\mathrm{H}-2 \mathrm{~L}^{\mathrm{d}}$ :peptide complex. The $\mathrm{H}-2 \mathrm{~L}^{\mathrm{d}}$ :Ig, loaded with given peptides, was preincubated with PE-conjugated anti-lgG1 mAb and used to stain transgenic spleen cells in conjunction with FITC-conjugated anti-CD8 mAb. (a) Characterization of transgenic mice expressing a TCR specific for the tumor antigen P1A. Spleen cells isolated from transgenic mice were stained either for coexpression of CD8 and the transgenic TCR (left panel) or for the specificity and sensitivity of dimer binding to the peptide dimer (center and right panels). (b) Dot plots depicting binding of the MHC:peptide complex to transgenic T cells. The peptide: $\mathrm{H}-2 \mathrm{~L}^{\mathrm{d}} \mathrm{Ig}$ complexes were used at 1:1 dilution, which consisted of $1 \mu \mathrm{g}$ of $\mathrm{H}-2 \mathrm{~L}^{\mathrm{d}} \mathrm{lg}$ and $0.75 \mu \mathrm{g}$ of peptides in $50 \mu \mathrm{l}$ PBS. (c) Quantitative comparison of the avidity of wild-type and mutant $\mathrm{H}-2 \mathrm{~L}^{\mathrm{d}}$ :P1A complex. Transgenic spleen cells were stained with given dilutions of $\mathrm{H}-2 \mathrm{~L}^{\mathrm{d}}$ loaded with excess wild-type P1A, mutant P1A, or control peptides. Data shown are mean fluorescence intensities of gated CD8 T cells. This experiment was repeated two to four times. Statistical significance was determined by paired $t$ test.

change of $\mathrm{T} \rightarrow \mathrm{G}$, resulting in the $\mathrm{P} 1 \mathrm{~A}(8 \mathrm{G})$ mutant, while the other had a mutation of $\mathrm{G} \rightarrow \mathrm{C}$, which leads to a substitution of $\mathrm{V}$ with $\mathrm{L}$ at $\mathrm{P} 8$, hereby called P8L. Since mutations at the P8 and P6 positions abolished $T$ cell recognition of $T$ cells, we conclude that antigenic drift also allows tumor evasion of polyclonal CTL responses, predominantly against the P1A epitope.

\section{Discussion}

Although antigenic drift has been established as a major mechanism for viral evasion of host immunity (1-5), its contribution to tumor evasion has not been systematically analyzed. There are at least three constraints that may have hindered progress in this area. First, given the rapid replication and - in the cases of RNA viruses and retroviruses - instability of the viral genome, an infected host can conceivably have a large number of mutant viral genomes available for immune selection. Second, perhaps because viruses are foreign to the host, immune responses are generally more robust than antitumor immunity, in terms of both the number of virus-specific CTLs $(20-22)$ and their effector function $(13,23)$. Third, in no experimental tumor model has the spectrum of tumor rejection antigens been fully characterized, despite the fact that many tumor antigens have been identified (24). The lack of clarity in the spectrum of tumor-rejection antigens makes it difficult to analyze mutations that may allow tumor evasion.

In order to separate these three intertwining issues, we used transgenic mice with either TCR $\alpha$ or TCR $\alpha \beta$ transgenes to determine whether tumors are genetically versatile enough to evade CTLs specific for a natural tumor antigenic epitope. Using T cells from mice with monoclonal ( $\alpha \beta$ chain-transgenic) and polyclonal ( $\alpha$ chain-transgenic) P1A-reactive T cells, we obtained clear evidence that mutations in at least four residues within a nine-AA peptide can each result in tumor evasion of a robust CTL response in vivo, as tumors with a single mutation were shown to recur and progressively grow in the presence of a strong CTL response.

It is of interest to note that of almost 70 clones of ex vivo tumor variants analyzed, apparent heterozygosity is found in only five clones. Since mutation in both alleles in the majority of the clones is unlikely, we believe the J558-Neo tumor cells probably have only one copy of the $P 1 A$ gene to begin with. Since P1A was recently mapped to the $\mathrm{X}$ chromosome (25), the hemizygosity of the gene may be due to the fact that the tumor cells are 
a
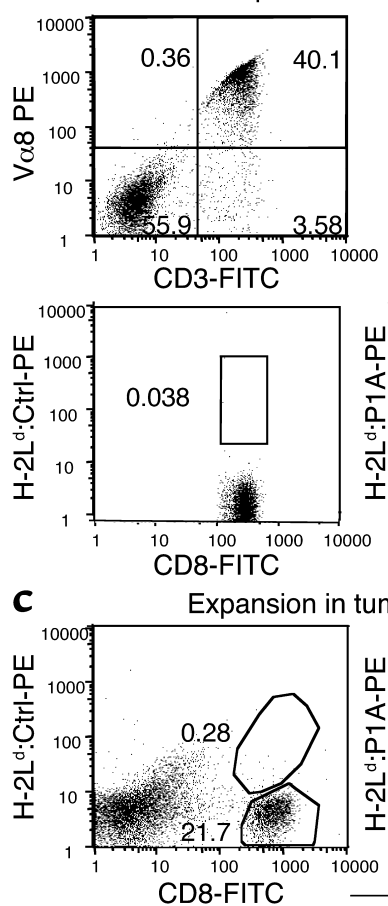

b

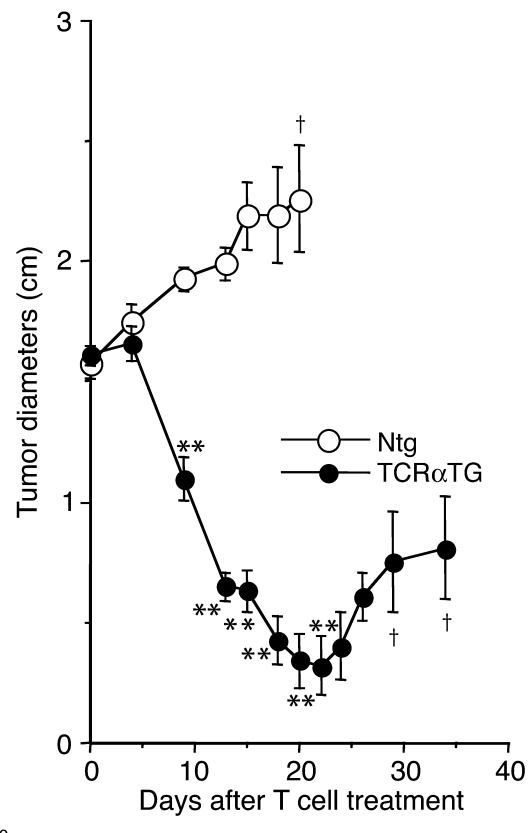

Figure 7

In adoptive therapy, TCR $\alpha$ chain-transgenic T cells mounted a robust CTL response against the P1A35-43 epitope presented by $\mathrm{H}-2 \mathrm{~L}^{\mathrm{d}}$ and caused transient rejection of large established tumors. (a) Expression of P1CTL TCR $\alpha$ chain on more than $90 \%$ of T cells, as revealed by coexpression of the transgenic $\mathrm{V} \alpha 8$ chain and CD3 or CD8. A significant (although small) proportion of CD8 T cells reacted specifically to $\mathrm{H}-2 \mathrm{~L}^{\mathrm{d}}$ :P1A complex. (b) Adoptive transfer therapy of mice with large tumor burdens using spleen cells from either TCR $\alpha$ chain-transgenic mice or their wild-type, nontransgenic $(\mathrm{Ntg})$ littermates. RAG-2-/- BALB/c mice were transplanted with $5 \times 10^{6} \mathrm{~J} 558-\mathrm{Neo}$ tumor cells. Once tumors were greater than $1.5 \mathrm{~cm}$ in diameter, unprimed spleen cells from either wild-type or transgenic littermates were injected intravenously. Tumor growth was monitored with calipers. Data show average tumor diameter of five mice per group. ${ }^{\dagger}$ The time of sacrifice. ${ }^{*}$ Significantly different from nontransgenic $(P \leq 0.01)$ as determined by Student $t$ test. (c) Robust CTL response in mice that received transgenic T cells. At 4-5 weeks after adoptive transfer, spleen cells of recipient mice were stained with anti-CD8 in conjunction with $\mathrm{H}-2 \mathrm{~L}^{\mathrm{d}}$ loaded with either control or P1A peptides. Three independent experiments revealed that $10-70 \%$ of CD8 T cells were capable of binding the $\mathrm{H}-2 \mathrm{~L}^{\mathrm{d}}$ :P1A complex.

derived from male mice (although our PCR analysis for a Y-chromosomal marker indicated that J558-Neo cells lack the Y chromosome, data not shown) or to the loss of heterozygosity of five clones during tumorigenesis. The apparent heterozygosity may be due to the accidental seeding of two independent clones into one well.

A related issue is whether the J558-Neo cells used for the current study are comprised of a mixture of cells with a high frequency of mutation in $P 1 A$ antigenic epitopes. We believe this is unlikely for three reasons. First, our analysis of 37 clones from parental cells by restriction enzyme mapping and sequencing revealed no mutations in the antigenic epitope. Second, our repeated efforts to select for P1A mutants with activated P1A-specific CTLs and parental tumor cells in ten 96 -well plates $\left(5 \times 10^{4}\right.$ tumor cells/well) have failed to obtain any CTL-resistant clones in vitro (data not shown). This may put the frequency of mutants at less than one per $5 \times 10^{7}$ cells. These results do suggest that cells with mutations in the P1A epitope are not present at high frequencies prior to $T$ cell selection. Since we started T cell therapy only after tumors had reached a size of more than $1.2 \mathrm{~cm}$ in diameter, we estimate that there may be up to $10^{9}$ tumor cells per mouse at the time of treatment. As a result, there could be a significant number of tumor variants for in vivo selection. Third, tumor cells isolated from $R A G-2^{-/-}$ mice that received no $T$ cell therapy did not reveal mutations in the P1A antigenic epitope (data not shown). Likewise, tumor cells recovered from mice that received nontransgenic $\mathrm{T}$ cells also contained no mutations in the P1A epitope. Therefore, the mutants described here must result from immune selection.

Our work demonstrated that, in addition to antigen loss (lack of expression of genes encoding tumor antigen) $(12,26)$ and MHC loss $(10,11)$, the antigenicity of tumors can be altered by antigenic drift. Thus, much like viruses, tumor cells can evade $T$ cell-mediated destruction through mutations in their antigenic epitopes. Although we are not aware of direct evidence, two lines of previous work are consistent with the notion that mutations in tumor antigenic epitopes can be a mechanism for immune evasion by tumor cells. First, CTL clones have long been used as tools to isolate tumor antigenic mutants in vitro, although long and repeated selection in vitro is usually needed for this purpose $(17,27,28)$. Second, 
a

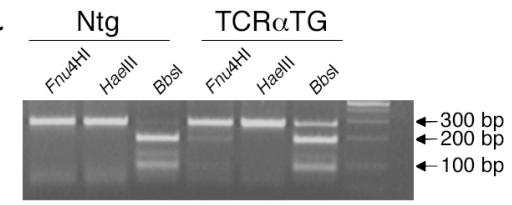

b

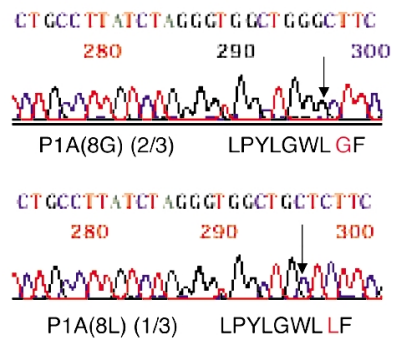

\section{Figure 8}

Tumors that recurred after TCR $\alpha$ transgenic T cell (TCR $\alpha \mathrm{Tg}$ ) therapy harbored multiple mutations in the P1A epitope. P1A gene fragments were amplified from recurrent tumors and analyzed by either restriction enzyme mapping or by cloning followed by sequencing. (a) Restriction mapping by a panel of restriction enzymes that identify mutations $\mathrm{P} 1 \mathrm{~A}(1 \mathrm{P})$ and $\mathrm{P} 1 \mathrm{~A}(7 \mathrm{P})$ (HaellI), $\mathrm{P} 1 \mathrm{~A}(6 \mathrm{R})($ Fnu4HI), and any alteration in nucleotides encoding P8 and P9 (Bbsl). P1A fragments were amplified from tumor cells from mice that received TCR $\alpha \mathrm{TG}$ wild-type $\mathrm{T}$ cell treatment (Ntg). Note the presence of mutation $\mathrm{P} 1 \mathrm{~A}(6 \mathrm{R})$ and alterations in $\mathrm{P} 8$ or P9 in the TCR $\alpha$ chain-transgenic group, but not in the nontransgenic group. Similar results were observed in another recurrent tumor from mice that received transgenic T cells (data not shown). (b) Sequencing of the P1A fragment that resisted digestion by $B b s l$. Note that of the three clones analyzed, two had mutation $\mathrm{P} 1 \mathrm{~A}(8 \mathrm{G})$ and one had mutation $\mathrm{P} 1 \mathrm{~A}(8 \mathrm{~L})$.

of the antigenic loss variants reported in the classic study (12), one was later proven to contain a mutation in the P1A epitope (position $7, \mathrm{~V} \rightarrow \mathrm{A}$ ) (17). This mutation prevented the clone from being recognized by one, but not another, CTL clone specific for the same epitope (17). However, the authors reported that the tumor clone harboring this mutation had a significant growth advantage in vitro over other tumor cells in the same isolate (12). It is thus unclear whether this tumor variant was selected for in vivo, and if so, whether it was selected based on its lack of antigenicity. If both these questions were answered in the affirmative, it would indicate that antigenic drift in tumors can occur in mice with an unmanipulated $\mathrm{T}$ cell repertoire.

Since the majority of our data involves adoptive transfer of purified transgenic $\mathrm{T}$ cells into immunedeficient mice, it is of interest to consider whether antigenic drift can occur in a normal tumor-bearing host in which the immune response consists of CD4 T cells, CD8 T cells, and B cells. Since we have verified our conclusion with adoptively transferred total spleen cells from TCR $\alpha$ chain-transgenic mice that have all subsets of lymphocytes, we believe antigenic drift can happen in a host with multiple subsets of lymphocytes. Moreover, recent studies indicate that homeostatic proliferation of $\mathrm{T}$ cells, upon adoptive transfer to an immune-deficient host, may enhance the efficacy of immune therapy both in experimental animals (29) and in humans (30). Such a setting is essentially identical to the approach in the current study.

Several properties of the P1A antigenic variants deserve comment. First, although there is a general correlation between the binding of the $\mathrm{H}-2 \mathrm{~L}^{\mathrm{d}}$ :peptide complex to the TCR and the ability of the peptides to induce $\mathrm{T}$ cell responses, the difference in binding is considerably less than that in biological function. For instance, mutation at $\mathrm{P} 6$ reduces binding by less than fourfold, yet it reduces the activity of the peptide in inducing proliferation and cytolysis by more than 10,000 -fold. The fact that a moderate difference in avidity of a TCR-ligand interaction has a drastic effect on the consequence of $\mathrm{T}$ cell recognition has been described and discussed extensively (31). Second, and more surprisingly, the intensity of MHC:peptide binding to a TCR does not always correlate well with biological function of the peptide. Thus $\mathrm{H}-2 \mathrm{~L}^{\mathrm{d}}: \mathrm{P} 1 \mathrm{~A}(7 \mathrm{P})$ binds much less efficiently than $\mathrm{H}-2 \mathrm{~L}^{\mathrm{d}}: \mathrm{P} 1 \mathrm{~A}(6 \mathrm{R})$ does, yet it is $\mathrm{P} 1 \mathrm{~A}(7 \mathrm{P})$, not $\mathrm{P} 1 \mathrm{~A}(6 \mathrm{R})$, that can induce $\mathrm{T}$ cell proliferation. One possible explanation is the higher potency of $\mathrm{P} 1 \mathrm{~A}(7 \mathrm{P})$ in binding $\mathrm{H}-2 \mathrm{~L}^{\mathrm{d}}$. Likewise, although $\mathrm{P} 1 \mathrm{~A}(6 \mathrm{R})$ and $\mathrm{P} 1 \mathrm{~A}(9 \mathrm{~L})$ have comparable binding to $\mathrm{H}-2 \mathrm{~L}^{\mathrm{d}}$ and TCR, there was at least a 100 -fold difference in biological activity between the two mutants. Davis et al. suggested that induced aggregation of MHC complex might be involved in T cell activation (32). In this scenario, different MHC:peptide complexes may differ in their ability to aggregate after TCR engagement, and the extent of such aggregation may determine the consequences of interaction between TCR and MHC:peptide. Finally, although some mutations do not completely abolish $\mathrm{T}$ cell recognition of the P1A peptide, cells harboring such mutations are completely resistant to CTL lysis. This is most likely due to limiting amounts of peptides produced intracellularly and explains the ability of these tumor mutants to evade $\mathrm{T}$ cell recognition.

Despite our extensive efforts, we have not been able to identify antigenic variants in the $P 1 A$ gene in recurrent tumors isolated from mice that received therapy with primed nontransgenic $\mathrm{T}$ cells (data not shown). However, we believe that this is due to our incomplete understanding of the nature of the $T$ cells responsible for tumor rejection. Better characterization of the antigenic spectrum will also allow determination of whether antigenic drift can lead to epitope spreading. Nevertheless, the results reported here reveal that $T$ cell therapy directed against a known antigenic epitope can be rendered ineffective by selection of tumor antigenic variants regardless of whether the $T$ cells are polyclonal or monoclonal. These results demonstrate that, much like viruses, tumor cells can evade $\mathrm{T}$ cell-mediated destruction through mutation in their antigenic epitope. Given the fact that a similar finding over 10 year ago (3) with transgenic $\mathrm{T}$ cells specific for one viral epitope has now been extended into a variety of in vivo models $(4,33,34)$, further characterization 
of tumor antigens should allow testing of whether the "antigenic drift" of tumor cells described here can be generalized to cancer patients. In this regard, it is worth noting that recent studies revealed the coexistence of tumor-reactive $T$ cells and tumors in cancer patients $(21,22)$, even in the draining lymph nodes (35). In many cases, the mRNA encoding tumor antigens was retained (35). It would be of interest to analyze whether the antigenic epitopes were mutated in these cancer patients. Regardless of whether tumor cells can evade $T$ cell responses to more than one epitope, the fact that mutations in a single epitope can occur suggests that vaccination based on a limited set of peptides (36) and gene therapy using TCR with monospecificity may have limited value (37).

Surprisingly, a recent study demonstrated that a mostly monoclonal $\mathrm{T}$ cell response develops over time, even if the initial $\mathrm{T}$ cells are polyclonal in nature (30). This may be overcome either by immunization with a multitude of tumor antigens (38-40) or by choosing those antigens that must be retained if the cancer cells are to remain cancerous (41).

\section{Acknowledgments}

We thank Yin Wang and Qunmin Zhou for valuable suggestions, and Jennifer Kiel and Lynde Shaw for editorial assistance. This work was supported by NIH grants CA-58033, AI-32981, and CA-69091 to Y. Liu, and by CA-82355 to P. Zheng.

1. Ciurea, A., et al. 2000. Viral persistence in vivo through selection of neutralizing antibody-escape variants. Proc. Natl. Acad. Sci. U. S. A. 97:2749-2754.

2. Parren, P.W., Moore, J.P., Burton, D.R., and Sattentau, Q.J. 1999. The neutralizing antibody response to HIV-1: viral evasion and escape from humoral immunity. AIDS. 13(Suppl. A):S137-S162.

3. Pircher, H., et al. 1990. Viral escape by selection of cytotoxic T cell-resistant virus variants in vivo. Nature. 346:629-633.

4. Goulder, P.J., et al. 2001. Evolution and transmission of stable CTL escape mutations in HIV infection. Nature. 412:334-338.

5. Ciurea, A., et al. 2001. CD4+ T-cell-epitope escape mutant virus selected in vivo. Nat. Med. 7:795-800.

6. Khong, H.T., and Restifo, N.P. 2002. Natural selection of tumor variants in the generation of "tumor escape" phenotypes. Nat. Immunol. 3:999-1005.

7. Wick, M., et al. 1997. Antigenic cancer cells grow progressively in immune hosts without evidence for $\mathrm{T}$ cell exhaustion or systemic anergy. J. Exp. Med. 186:229-238.

8. Ochsenbein, A.F., et al. 1999. Immune surveillance against a solid tumor fails because of immunological ignorance. Proc. Natl. Acad. Sci. U. S. A. 96:2233-2238.

9. Shrikant, P., Khoruts, A., and Mescher, M.F. 1999. CTLA-4 blockade reverses CD8+ T cell tolerance to tumor by a CD4+ T cell- and IL-2dependent mechanism. Immunity. 11:483-493.

10. Zheng, P., et al. 1998. Proto-oncogene PML controls genes devoted to MHC class I antigen presentation. Nature. 396:373-376.

11. Seliger, B., Maeurer, M.J., and Ferrone, S. 1997. TAP off-tumors on. Immunol. Today. 18:292-299.

12. Uyttenhove, C., Maryanski, J., and Boon, T. 1983. Escape of mouse mastocytoma P815 after nearly complete rejection is due to antigen-loss variants rather than immunosuppression. J. Exp. Med. 157:1040-1052.

13. Sarma, S., et al. 1999. Cytotoxic T lymphocytes to an unmutated tumor antigen P1A: normal development but restrained effector function. J. Exp. Med. 189:811-820.

14. Bai, X.F., et al. 2001. Local costimulation reinvigorates tumor-specific cytolytic T lymphocytes for experimental therapy in mice with large tumor burdens. J. Immunol. 167:3936-3943.
15. Liu, X., et al. 2001. B7H costimulates clonal expansion of, and cognate destruction of tumor cells by, $\mathrm{CD}^{+} \mathrm{T}$ lymphocytes in vivo. J. Exp. Med. 194:1339-1348.

16. Smith, J.D., Myers, N.B., Gorka, J., and Hansen, T.H. 1993. Model for the in vivo assembly of nascent Ld class I molecules and for the expression of unfolded Ld molecules at the cell surface. J. Exp. Med. 178:2035-2046.

17. Lethe, B., van den Eynde, B., van Pel, A., Corradin, G., and Boon, T. 1992. Mouse tumor rejection antigens P815A and P815B: two epitopes carried by a single peptide. Eur. J. Immunol. 22:2283-2288.

18. Evavold, B.D., Sloan-Lancaster, J., and Allen, P.M. 1993. Tickling the TCR: selective T-cell functions stimulated by altered peptide ligands. Immunol. Today. 14:602-609.

19. Hogquist, K.A., et al. 1994. T cell receptor antagonist peptides induce positive selection. Cell. 76:17-27.

20. Butz, E.A., and Bevan, M.J. 1998. Massive expansion of antigen-specific CD8+ T cells during an acute virus infection. Immunity. 8:167-175.

21. Lee, P.P., et al. 1999. Characterization of circulating T cells specific for tumor-associated antigens in melanoma patients. Nat. Med. 5:677-685.

22. Romero, P., et al. 1998. Ex vivo staining of metastatic lymph nodes by class I major histocompatibility complex tetramers reveals high numbers of antigen- experienced tumor-specific cytolytic T lymphocytes. J. Exp. Med. 188:1641-1650.

23. Ramarathinam, L., Castle, M., Wu, Y., and Liu, Y. 1994. T cell costimulation by B7/BB1 induces CD8 $\mathrm{T}$ cell-dependent tumor rejection: an important role of B7/BB1 in the induction, recruitment, and effector function of antitumor T cells. J. Exp. Med. 179:1205-1214.

24. Boon, T., Cerottini, J.C., Van den Eynde, B., van der Bruggen, P., and Van Pel, A. 1994. Tumor antigens recognized by T lymphocytes. Annu. Rev. Immunol. 12:337-365.

25. Gregory, S.G., et al. 2002. A physical map of the mouse genome. Nature. 418:743-750.

26. Dudley, M.E., and Roopenian, D.C. 1996. Loss of a unique tumor antigen by cytotoxic $\mathrm{T}$ lymphocyte immunoselection from a 3-methylcholanthrene-induced mouse sarcoma reveals secondary unique and shared antigens. J. Exp. Med. 184:441-447.

27. Tanaka, Y., and Tevethia, S.S. 1988. In vitro selection of SV40 T antigen epitope loss variants by site-specific cytotoxic $\mathrm{T}$ lymphocyte clones. J. Immunol. 140:4348-4354.

28. Lill, N.L., Tevethia, M.J., Hendrickson, W.G., and Tevethia, S.S. 1992. Cytotoxic T lymphocytes (CTL) against a transforming gene product select for transformed cells with point mutations within sequences encoding CTL recognition epitopes. J. Exp. Med. 176:449-457.

29. Dummer, W., et al. 2002. T cell homeostatic proliferation elicits effective antitumor autoimmunity. J. Clin. Invest. 110:185-192. doi:10.1172/ JCI200215175.

30. Dudley, M.E., et al. 2002. Cancer regression and autoimmunity in patients after clonal repopulation with antitumor lymphocytes. Science. 298:850-854

31. Jameson, S.C., Hogquist, K.A., and Bevan, M.J. 1995. Positive selection of thymocytes. Annu. Rev. Immunol. 13:93-126.

32. Reich, Z., et al. 1997. Ligand-specific oligomerization of T-cell receptor molecules. Nature. 387:617-620.

33. McMichael, A. 1998. T cell responses and viral escape. Cell. 93:673-676.

34. Borrow, P., and Shaw, G.M. 1998. Cytotoxic T-lymphocyte escape viral variants: how important are they in viral evasion of immune clearance in vivo? Immunol. Rev. 164:37-51.

35. Romero, P., et al. 1998. Ex vivo staining of metastatic lymph nodes by class I major histocompatibility complex tetramers reveals high numbers of antigen-experienced tumor-specific cytolytic T lymphocytes. J. Exp. Med. 188:1641-1650.

36. Coulie, P.G., et al. 2001. A monoclonal cytolytic T-lymphocyte response observed in a melanoma patient vaccinated with a tumor-specific antigenic peptide encoded by gene MAGE-3. Proc. Natl. Acad. Sci. U. S. A. 98:10290-10295.

37. Stanislawski, T., et al. 2001. Circumventing tolerance to a human MDM2derived tumor antigen by TCR gene transfer. Nat. Immunol. 2:962-970.

38. Boczkowski, D., Nair, S.K., Nam, J.H., Lyerly, H.K., and Gilboa, E. 2000. Induction of tumor immunity and cytotoxic T lymphocyte responses using dendritic cells transfected with messenger RNA amplified from tumor cells. Cancer Res. 60:1028-1034.

39. Banchereau, J., et al. 2001. Immune and clinical responses in patients with metastatic melanoma to $\mathrm{CD} 34(+)$ progenitor-derived dendritic cell vaccine. Cancer Res. 61:6451-6458.

40. Srivastava, P.K., and Udono, H. 1994. Heat shock protein-peptide complexes in cancer immunotherapy. Curr. Opin. Immunol. 6:728-732.

41. Beck-Engeser, G.B., et al. 2001. Point mutation in essential genes with loss or mutation of the second allele: relevance to the retention of tumorspecific antigens. J. Exp. Med. 194:285-300. 